hep-th/0703162

March 2007

\title{
Entropy of Contracting Universe in Cyclic Cosmology
}

\author{
Lauris Baum and Paul H. Frampton \\ Department of Physics and Astronomy, \\ University of North Carolina at Chapel Hill, NC 27599-3255, USA
}

\begin{abstract}
Following up a recent proposal [1] for a cyclic model based on phantom dark energy, we examine the content of the contracting universe (cu) and its entropy $S_{c u}$. We find that beyond dark energy the universe contains on average zero or at most a single photon which if present immediately after turnaround has infinitesimally energy which subsequently blue shifts to produce $e^{+} e^{-}$pairs. These statements are independent of the equation of state $\omega=p / \rho$ of dark energy provided $\omega<-1$. Thus $S_{c u}=0$ and if observations confirm $\omega<-1$ the entropy problem is solved. We discuss the absence of a theoretical lower bound on $\phi=|\omega+1|$, then describe an anthropic fine tuning argument that renders unlikely extremely small $\phi$. The present bound $\phi \lesssim 0.1$ already implies a time until turnaround of $\left(t_{T}-t_{0}\right) \gtrsim 100 \mathrm{~Gy}$.
\end{abstract}


In a recent paper [1] we suggested a cyclic model which solves the entropy problem [2]. Technical calculations are certainly desirable to demonstrate the consistency of our model but we know of no fatal flaw. The most important new ingredient is the idea that the contracting universe has essentially zero entropy and comes back empty of matter. Here we take the model seriously \#1 and examine more critically some general features including its possibilty of being tested.

The contracting universe of the cyclic model contains dark energy with zero entropy and possibly, as was only generally stated in [1], a small amount of radiation which could possess entropy. The deflation at turnaround reduces entropy from a gigantic value $\mathrm{O}(>$ $10^{88}$ ) to an extremely low value there cited as $\mathrm{O}\left(10^{1}\right)$. An unrealistic value for the dark energy equation of state $\omega=p / \rho=-4 / 3$ was employed simply for algebraic simplicity as it makes $\rho_{\Lambda} \propto a$, and no attempt was made at a realistic description of our universe. In the present article, we shall study the entropy of the contracting universe in this speculative scenario more quantitatively and now will use arbitrary $\omega=-1-\phi$ with $\phi>0$ so that $\rho_{\Lambda} \propto a^{3 \phi}$.

The quantity $\phi$ is the most important parameter for observational discrimination between this cyclic model and a cosmological constant \#2. The next test of $\phi \neq 0$ will likely come from the Planck Surveyor satellite [7]. One wonders, therefore, how different from zero $\phi$ is? There is no lower bound on $\phi$ to make the model work except that it must be non zero. We already know $\phi \lesssim 0.1$ from the WMAP3 data [8]. If $\phi$ is truly infinitesimal, the test must await improved technology. To restore optimism we shall, at the end of the Letter, describe an anthropic fine tuning argument that shows that extremely small $\phi$ is unlikely.

In [1], it was emphasized that the universe comes back empty of matter including black holes. The presence of matter during contraction causes apparently insuperable problems because accelerated structure formation will precipitate a premature bounce. Black holes, if present, will expand and proliferate with the same consequence. But the presence of radiation must also be carefully studied because although at turnaround the photon energy is infinitesimal $\left(E_{\gamma} \lesssim 10^{-200} \mathrm{eV}\right.$ ), the blue shifting during contraction leads before the bounce to production of $e^{+} e^{-}$pairs, undesirable because generically they will create problems with continued contraction. As we shall show herein there are fortunately no photons in the contracting phase of the cycle, only the truly innocuous dark energy.

The cyclic model contains one free parameter, the common density $\rho_{C}$ at which the universe both turns around and bounces. Since the bounce is independent of $\omega$ we begin with it and take as bounce temperatures $T_{B}=10^{p} \mathrm{GeV}$ with, to be above the weak and below the Planck scales, $3 \leq p \leq 17$. Using the derivation in [1] this gives $\rho_{C}=\eta \rho_{\mathrm{H}_{2} \mathrm{O}}$ where $\eta=10^{(19+4 p)}$ and $\rho_{H_{2} 0}=1 \mathrm{~g} / \mathrm{cm}^{3}$ is the density of water, an easily imaginable

\footnotetext{
\#1 It has been said the problem is not that theoretical physicists take their own model too seriously but that they do not take it seriously enough [3].

\#2 and from the Steinhardt-Turok cyclic model [4-6].
} 
unit somewhere between the unimaginably small present mean cosmic density and the unimaginably large critical density $\rho_{C}$ at turnaround and bounce.

Going now to the turnaround at time $t=t_{T}$ the scale factor $a\left(t_{T}\right)$ is given by (since $a\left(t_{0}\right)=1$ and putting $\left.\rho_{0}=10^{-29} \rho_{\mathrm{H}_{2} \mathrm{O}}\right)$

$$
a\left(t_{T}\right)^{3 \phi}=10^{29} \eta=10^{48+4 p}
$$

The present radiation temperature is $\left(T_{\gamma}\right)_{0}=2 \times 10^{-4} \mathrm{eV}$, and so from Eq.(1) the radiaition temperature at turnaround is

$$
\left(T_{\gamma}\right)_{T}=2 \times 10^{-4}\left(10^{(48+4 p)}\right)^{-1 / 3 \phi} \mathrm{eV}
$$

which is infinitesimal: putting $\phi=0.1$, Eq.(2) gives $10^{-200} \mathrm{eV}$ for $\mathrm{p}=3$ and $10^{-390} \mathrm{eV}$ for $\mathrm{p}=17$; with $\phi=0.01$, the photon energy is $10^{-2000} \mathrm{eV}$ for $\mathrm{p}=3$ and $10^{-3900} \mathrm{eV}$ for $\mathrm{p}=17$. In all cases, the photon wavelength is an astronomical number of orders of magnitude longer than the present Hubble length.

To evaluate the contraction entropy we need to estimate many such photons are in one causal patch at turnaround. As shown in [1] the deflationary factor multiplying entropy at turnaround must be much less than the inverse of the inflationary increase $\left(\gtrsim 10^{84}\right)$ of the early universe. We take the huge number of causal patches to be $10^{90} \alpha$ where $\alpha \gg 1$ is a parameter to allow an arbitrarily larger number, and $\alpha=1$ will give an overestimate of contraction entropy.

At turnaround the scale factor is

$$
a\left(t_{T}\right)=\left(10^{(48+4 p)}\right)^{\frac{1}{3 \phi}}
$$

so taking the present volume as $10^{84} \mathrm{~cm}^{3}$ and the present radiation density as $\rho_{r}\left(t_{0}\right)=$ $10^{-33} \mathrm{~g} / \mathrm{cm}^{3}=1 \mathrm{eV} / \mathrm{cm}^{3}$ gives for the radiation energy in one causal patch

$$
\left(E_{r}\right)_{\text {patch }}=\frac{1}{(100 \alpha)^{3}}\left(10^{(48+4 p)}\right)^{-\frac{1}{3 \phi}} \mathrm{eV}
$$

Comparison with Eq.(2) then gives for the number of photons per causal patch

$$
n_{\gamma}=\frac{1}{200 \alpha^{3}} \ll 1
$$

which is small even for the unrealistic case $\alpha=1$ and essentially zero for $\alpha \gg 1$. Thus, the entropy of the contracting universe $(\mathrm{cu})$ vanishes $S_{c u}=0$ for any value of equation of state of the dark energy $\omega=p / \rho=-1-\phi$ since Eq.(5) has no $\phi$ dependence. 


\section{Anthropic fine tuning argument about $\phi$}

The time until turnaround is given, e.g. [9], by

$$
\left(t_{T}-t_{0}\right) \simeq \frac{t_{0}}{\phi}
$$

so if we take, for simplicity, the origin of life to have occurred at $t_{0}$ after the most recent bounce we see from Eq. (6) that given small $\phi \ll 1$ then $\phi$ measures the fraction of the expansion phase taken to originate life. An anthropic argument is: it is unreasonable for the fraction $\phi$, assuming it is non zero, to be extremely close to zero.

The special case $\phi=0$ is the standard cosmological model with a cosmological constant where there is no turnaround and the future lifetime is infinite so the origin of life necessarily takes place after a vanishing fraction of the expansion lifetime. Although such an infinite expansion seems to us unaesthetic [1], not all colleagues share our concern.

As soon as one commits to $\phi \neq 0$, however, the anthropic type argument emerges and it is unlikely that $\phi<<<1$. For example, if $\phi=10^{-3}$ the length of the expansion phase is $10^{4}$ Gy whereas life orinated after only about 10 Gy which is only $0.1 \%$ of the expansion time. If life plays a central role in our universe, as in our understanding is the spirit of the anthropic principle, such a tiny value of $\phi$ is strongly disfavored; one expects at least $\phi \gtrsim 0.01$ so the fraction before the origin of life is $\gtrsim 1.0 \%$ of the total expansion time.

This encouraging argument makes it more optimistic that the next generation of observations such as the Planck Surveyor [7] will succeed in detecting a $\phi \neq 0$.

\section{Acknowledgements}

This work was supported in part by the U.S. Department of Energy under Grant No. DE-FG02-06ER41418. 


\section{References}

[1] L. Baum and P.H. Frampton, Phys. Rev. Lett. 98, 071301 (2007). hep-th/0610213.

[2] R.C. Tolman, Relativity, Thermodynamics and Cosmology. Oxford University Press (1934) reprinted by Dover Books.

[3] S. Weinberg, private communication.

[4] P.J. Steinhardt and N. Turok, Science 296, 1436 (2002).

[5] P.J. Steinhardt and N. Turok, Phys. Rev. D65, 126003 (2002). hep-th/0111098.

[6] P.J. Steinhardt and N. Turok. astro-ph/0605173.

[7] http://www.rssd.esa.int/index.php?project=PLANCK

[8] D.N. Spergel, et al, Wilkinson Microwave Anisotropy Probe (WMAP) Three Year Results: Implications for Cosmology. (March 17, 2006). astro-ph/0603449

L. Page, et al, Wilkinson Microwave Anisotropy Probe (WMAP) Three Year Results: Polarization reults. (March 17, 2006). astro-ph/0603450

G. Hinshaw, et al, Wilkinson Microwave Anisotropy Probe (WMAP) Three Year Results: Temperature results. (March 17, 2006). astro-ph/0603451 http://map.gsfc.nasa.gov/m_mm/pub_papers/threeyear.html

[9] P.H. Frampton and T. Takahashi, Phys. Lett. B557, 135 (2003). astro-ph/0211544. 